\title{
Estresse oxidativo na interrupção da ventilação mecânica em pacientes vítimas de traumatismo cranioencefálico: um estudo randomizado e cego
}

\author{
Oxidative stress in the interruption of mechanical ventilation \\ in patients with traumatic brain injury: a randomized, blind study
}

Anderson Antunes da Costa Moraes ${ }^{1,2}$, Luã Alves Araujo1,2, Rodrigo Alcântara Carnevalli de Araújo², Rodrigo Santiago Barbosa Rocha ${ }^{3}$ Leonardo Ramos Nicolau da Costa ${ }^{4}$, Kéven Lorena de Paula Gonçalves², Gabriela Martins de Lima ${ }^{2,5}$

IUniversidade do Estado do Pará (UEPA) - Belém (PA), Brasil.

${ }^{2}$ Hospital Metropolitano de Urgência e Emergência (HMUE) - Belém (PA), Brasil.

${ }^{3}$ Ciências do Movimento Humano, Universidade Metodista de Piracicaba (UNIMEP) - Piracicaba (SP), Brasil.

${ }^{4}$ Ensino e Pesquisa do Hospital Metropolitano de Urgência e Emergência (HMUE) - Belém (PA), Brasil.

${ }^{5}$ Programa de Pós Graduação Ensino e Saúde na Amazônia (UEPA) - Belém (PA), Brasil.

DOI: http://dx.doi.org/10.7322/abcshs.v43i3.1118

\begin{abstract}
RESUMO
Introdução: Os efeitos tóxicos do oxigênio não estão bem estabelecidos em seres humanos. Objetivo: Analisar os níveis de estresse oxidativo (EOx) na interrupção da ventilação mecânica (VM) em pacientes vítimas de TCE. Métodos: Estudo prospectivo, longitudinal, analítico, randomizado e cego. Participaram 12 pacientes: PSV $(n=6)$ e grupo Tubo-T $(n=6)$. A interrupção da VM deu-se em: PSV- $\left(\triangle 7 \mathrm{cmH}_{2} \mathrm{O}\right)$ e PEEP (5 $\mathrm{CmH}_{2} \mathrm{O}$ ) durante 30 minutos; Tubo- $\mathrm{T}$ - o paciente foi conectado ao tubo $\mathrm{T}$ com FiO2 a 0,4 durante 30 minutos. Realizou-se avaliação morfológica do EOx no plasma sanguíneo pelo Dry layer oxidative test. A coleta foi realizada: com 24h de VM, antes do teste, em 15' de teste e após o teste PSV ou Tubo-T. Para análise da normalidade utilizou-se o teste de Shapiro-Wilk para análise intragrupo e intergrupos, o teste ANOVA seguido de Tukey; para correlaçãoCorrelação de Spearman ( $p$ valor 5\%). Resultados: Não houve aumento estatisticamente significativo na descontinuidade da matriz extracelular nos grupos ( $p>0,05)$. Os níveis de EOx encontraramse elevados (grau III-moderado) nas primeiras avaliações. Não foi encontrada correlação significativa entre os parâmetros de VM com o EOx. O grupo PSV permaneceu em média 12.33 dias sob VM, tendo EOx de 29.02 (grau III/ moderado) - $r=0.8, p=0.01$; o grupo Tubo T em média 12.83 \pm 2.2 dias, tendo EOx de 31.03 \pm 5.41 (grau IV/grave)- $r=0.9, p=0.07$. Conclusão: Não houve diferença no comportamento do EOx entre os métodos de interrupção da VM. No entanto, o EOx aumentou com o tempo de VM.
\end{abstract}

Palavras-chave: traumatismos craniocerebrais; estresse oxidativo; respiração artificial.

\begin{abstract}
Introduction: The toxic effects of oxygen are not well established in human. Objective: To analyze the oxidative stress levels (EOX) in the interruption of mechanical ventilation (MV) in PSV and T-tube in patients with TBI. Methods: prospective, longitudinal, analytical, randomized and blind study. Twelve patients were assigned to PSV $(n=6)$; T-tube $(n=6)$ groups. The MV interruption occurred in: PSV- $(\triangle 7 \mathrm{cmH} 2 \mathrm{O})$ and PEEP $(5 \mathrm{cmH} 2 \mathrm{O})$. T-tube the patient was connected to the T-tube with $0.4 \mathrm{FiO} 2$ for 30 minutes. Morphological evaluation of EOx in the blood plasma was performed by the Dry layer oxidative test. The collection was performed: with $24 \mathrm{~h}$ of MV, before the test, in 15 'of test and after the PSV or T-tube. For the analysis of normality, the ShapiroWilk test was used; for intragroup analysis and intergroups, the ANOVA test followed by Tukey; for correlation the Spearman correlation - $p$ value 5\%. Results: There was no statistically significant increase in the extracellular matrix discontinuity in the groups ( $p>0.05)$. EOx levels were elevated (grade IIImoderate) in the first evaluations. No significant correlation was found between the VM parameters and the EOx. The PSV group remained on average 12.33 days under MV, with EOx of 29.02 (grade $\mathrm{II} /$ moderate) $-\mathrm{r}=0.8, \mathrm{p}=0.01$; the $\mathrm{T}$-tube group averaged $12.83 \pm 2.2$ days, with EOx of $31.03 \pm 5.41$ (grade IV/severe) $r=0.9, p=0.07$. Conclusion: there was no difference in the EOx behavior between the methods of MV interruption. However, the EOx increased with the VM time.
\end{abstract}

Keywords: Craniocerebral trauma; oxidative stress: respiration, artificial.

Recebido em: 14/02/2018

Revisado em: 05/07/2018

Aprovado em: 05/07/2018

Autor para correspondência: Anderson Antunes da Costa Moraes - Hospital Metropolitano de Urgência e Emergência no Trauma - BR 316 , s/n, Km 3 Bairro Atalaia - CEP: 67010-000 - Ananindeua (PA), Brasil - E-mail: antunesanderson@hotmail.com.

Conflito de interesses: nada a declarar. 


\section{INTRODUÇÃO}

A importância do Traumatismo crânio-encefálico (TCE) se dá pela sua ocorrência: cerca de 200 a 300 pessoas a cada 100.000 habitantes são hospitalizadas em virtude da lesão craniana, sendo 3 a 4 vezes mais casos examinados de urgência sem necessidade de hospitalização. No entanto, muitos desses pacientes evoluem com insuficiência respiratória necessitando de intubação traqueal e suporte ventilatório artificial ${ }^{1}$.

A ventilação mecânica invasiva (VMI) é um método que oferece pressão positiva nas vias aéreas por meio de uma prótese para o tratamento de pacientes com insuficiência respiratória aguda (IRpA) ou crônica agudizada. Ela tem por objetivos manter as trocas gasosas; diminuir o trabalho da musculatura respiratória, reduzir o desconforto respiratório e permitir a aplicação de terapêuticas específicas ${ }^{2,3}$.

O suporte ventilatório invasivo é utilizado em cerca de $40 \%$ dos pacientes admitidos nas unidades de terapia intensiva (UTI), dos quais grande parte necessita de ventilação mecânica (VM) prolongada, estando susceptíveis às complicações associadas ao seu uso como: pneumonia associada à ventilação mecânica, fraqueza muscular respiratória e periférica, produção de espécies reativas de oxigênio, entre outras ${ }^{4}$. Com a melhora do quadro clínico do paciente, inicia-se então a retirada da VM, também denominada de desmame ventilatório ${ }^{1}$.

No processo de desmame ventilatório são utilizados vários recursos, entre eles: o método ventilação por pressão de suporte (PSV), que pode resultar em menor taxa de insucesso do desmame, se comparado ao desmame com períodos progressivos (30 a $120 \mathrm{mi}$ nutos) de respiração espontânea com tubo- $\mathrm{T}^{1,3,5}$. Nesse sentido, embora a quantidade de pesquisas a respeito seja ainda muito restrita, entende-se que uma das complicações envolvidas na ventilação mecânica é o estresse oxidativo ${ }^{6}$. Esse processo ocorre devido ao desequilíbrio entre a produção e a neutralização de agentes oxidantes no organismo, propiciando a produção excessiva ou o detrimento da velocidade de remoção dos radicais livres. Adiciona-se a esse processo, a oxidação de biomoléculas que culmina com a perda de funções biológicas e/ou desequilíbrio homeostático, cuja manifestação é o dano oxidativo potencial nas células e tecidos ${ }^{7,8}$.

Os efeitos tóxicos do oxigênio ainda não estão bem estabelecidos em seres humanos, mas quando administrado em altas doses ou por um período prolongado de tempo, o oxigênio pode causar lesões pulmonares e sistêmicas ${ }^{2,9}$.

Em um estudo ${ }^{10}$, foi avaliado o dano oxidativo e processos inflamatórios no pulmão e no diafragma de camundongos frente à exposição à fumaça do cigarro que contem alta quantidade de agentes oxidantes. Nesse estudo, demonstrou-se que a exposição à fumaça do cigarro ocasiona processo oxidativo tanto no tecido pulmonar quanto no tecido muscular, tendo um efeito adicional no músculo respiratório.

Diante do exposto, entende-se os efeitos deletérios do estresse oxidativo no sistema respiratório. No entanto, há a necessidade de maiores evidências quanto ao comportamento do estresse oxidativo frente à interrupção do suporte ventilatório em PSV ou Tubo T, e, dessa forma, vislumbrar mais um parâmetro para a realização de um manejo correto na descontinuação do suporte ventilatório de pacientes vítimas de TCE submetidos à VM na UTI.

Diante disso, o objetivo da pesquisa foi analisar os níveis de estresse oxidativo na interrupção da ventilação mecânica em PSV e Tubo $\mathrm{T}$ em pacientes vítimas de TCE.

\section{MÉTODOS}

Esta pesquisa foi aprovada pelo Comitê de ética e pesquisa da Universidade do Estado do Pará sob o número CAEE 65662217.9.0000.5174, respeitando as normas de pesquisa envolvendo seres humanos (Res. CNS 466/12) do conselho nacional de saúde. Este estudo foi do tipo prospectivo, longitudinal, analítico, randomizado e cego, sendo realizado nas Unidades de Terapia Intensiva Adulto do Hospital Metropolitano de urgência e Emergência (HMUE), Ananindeua, Pará, Brasil. A coleta de dados foi realizada entre março de 2017 e setembro de 2017.

A casuística foi composta por 12 pacientes, vítimas de TCE internados nas Unidades de Terapia Intensiva (1 e 2) do Hospital Metropolitano de Urgência e Emergência. Foram incluídos na pesquisa pacientes vítimas de TCE de ambos os sexos, maiores de 18 anos, hemodinamicamente estáveis, em ventilação mecânica invasiva com tubo orotraqueal; e que tiveram o TCLE assinado pelo responsável.

Por outro lado, adotou-se como critérios de exclusão pacientes com doenças de má formação arterio-venosas ou oncológicas associadas; em suplementação de antioxidantes; que receberem transfusão sanguínea em até $72 \mathrm{~h}$ antes da coleta; pacientes com distúrbios de coagulação; que realizaram hemodiálise; e que evoluíram a óbito antes do sucesso ou falha da interrupção da ventilação mecânica; que foram transferidos para outros serviços; e pacientes que evoluíram para cânula de traqueostomia.

Os pacientes foram avaliados de acordo com as variáveis contidas na Ficha de avaliação elaborada pelo autor, sendo estes o registro hospitalar (RH), data de admissão na UTI, data de avaliação, idade, sexo, mecanismo do trauma, tipo de TCE, tipo de tratamento, parâmetros do suporte ventilatório invasivo ( $\mathrm{PEEP}, \mathrm{FiO} 2)$; gasometria arterial ( $\mathrm{PH}, \mathrm{PaO} 2, \mathrm{PaCO} 2, \mathrm{HCO} 3, \mathrm{BE}, \mathrm{SO} 2 \%)$, relação $\mathrm{PaO} 2 / \mathrm{FiO} 2$ e tempo de ventilação mecânica. Após isso, os pacientes foram distribuídos entre o grupo PSV ( $\mathrm{n}=6)$ e grupo Tubo-T $(\mathrm{n}=6)$, por meio do software Random Number Generator Pro. A interrupção da ventilação mecânica foi realizada de acordo com cada grupo. No grupo PSV foi realizada com pressão de suporte $\left(\Delta 7 \mathrm{cmH}_{2} \mathrm{O}\right)$ mais pressão positiva ao final da expiração $\left(5 \mathrm{cmH}_{2} \mathrm{O}\right)$ durante $30 \mathrm{mi}$ nutos. Já no grupo Tubo-T o paciente ventilou por meio do tubo orotraqueal, conectado a uma peça em forma de " $\mathrm{T}$ " com suporte de oxigênio ( $\mathrm{FiO} 20,4$ ) durante 30 minutos.

Após isso, foi realizada a avaliação morfológica do estresse oxidativo no plasma sanguíneo pelo teste denominado de Microscopia Ótica para a Análise Celular In Vitro; ou Teste HLB. O teste também pode ser chamado de Dry layer oxidative test. A coleta foi realizada 
pelo próprio pesquisador por meio de uma punção capilar, como os testes realizados na glicemia, na face medial da polpa digital do quarto dedo da mão previamente higienizada com álcool, sendo coletada uma gota de sangue em uma lâmina de microscopia por meio de cinco toques desta lâmina junto à gota ${ }^{11}$. Após este procedimento, a lâmina foi armazenada em caixas próprias para lâminas e transportada pelo pesquisador. As coletas foram realizadas em até 24 h pós admissão na UTI; imediatamente antes do teste de interrupção (PSV ou Tubo-T); 15 minutos após o início do teste; e imediatamente após o término do teste.

Ao final deste período, o padrão morfológico deste coágulo foi microscopicamente observado sob um aumento de 40 vezes em microscópio óptico da marca Nikon ${ }^{\circledR}$ modelo Binocular E200 Standard. A respectiva graduação qualitativa será registrada por câmera fotográfica acoplada da marca Nikon ${ }^{\circledast}$ em uma planilha eletrônica para posterior análise. A observação dos padrões morfológicos dos diferentes coágulos obtidos foi realizada em 1 imagem obtida em cada uma das lâminas e a avaliação foi realizada através do programa denominado Image Pro Plus $4.0^{\circledR}$, por um pesquisador cego que não obtinha conhecimento sobre a qual grupo cada paciente pertencia, o qual recebeu as imagens nomeadas por códigos para que realizasse a análise da descontinuidade da matriz extracelular e posteriormente a classificação do estresse oxidativo.

Foi analisada a mensuração da descontinuidade de área da matriz extracelular de cada campo fotográfico, sendo tabuladas em uma planilha no Excel $^{\circledR}$ para a análise estatística. O teste proporciona análise indireta do estresse oxidativo no plasma sanguíneo, graduando a área descontínua em graus: grau I (normal) - área de descontinuidade da matriz extracelular menor que 10\%; grau II (leve) - área de descontinuidade entre $10 \%$ e $20 \%$; grau III (moderado) - área de descontinuidade entre $20 \%$ e $30 \%$; grau IV (grave) área de descontinuidade da matriz extracelular variando entre $30 \%$ e $40 \%$; grau V (muito grave) com área de descontinuidade maior que $40 \%{ }^{11}$.

As avaliações foram tabuladas em uma planilha do Excel $^{\circledR}$. Em seguida, foi utilizado para análise dos dados o programa BioEstat ${ }^{\circledR}$ 5.3. Para análise da normalidade das variáveis utilizou-se o teste de Shapiro-Wilk. Para análise da variância dos dados intragrupos e intergrupos utilizou-se o teste ANOVA seguido de Tukey. Para a correlação de variáveis utilizou-se a correlação de Spearmam. O nível de rejeição da hipótese de nulidade ficou estabelecido em 0,05 ou $5 \%$.

\section{RESULTADOS}

O universo amostral compreendeu 39 pacientes vítimas de TCE sob ventilação mecânica. Foram excluídos 25 pacientes que evoluíram para cânula de traqueostomia; e 2 pacientes que evoluíram à óbito. Foram incluídos na pesquisa 12 pacientes, que foram subdivididos em dois grupos: Grupo PSV com 6 pacientes, sendo $5(83,33 \%)$ do sexo masculino e $1(16,66 \%)$ do sexo feminino; e Grupo Tubo-T também com 6 pacientes, sendo 5 (83,33\%) do sexo masculino e $1(16,66 \%)$ do sexo feminino. O fluxo de pacientes incluídos no estudo é demonstrado na Figura 1.

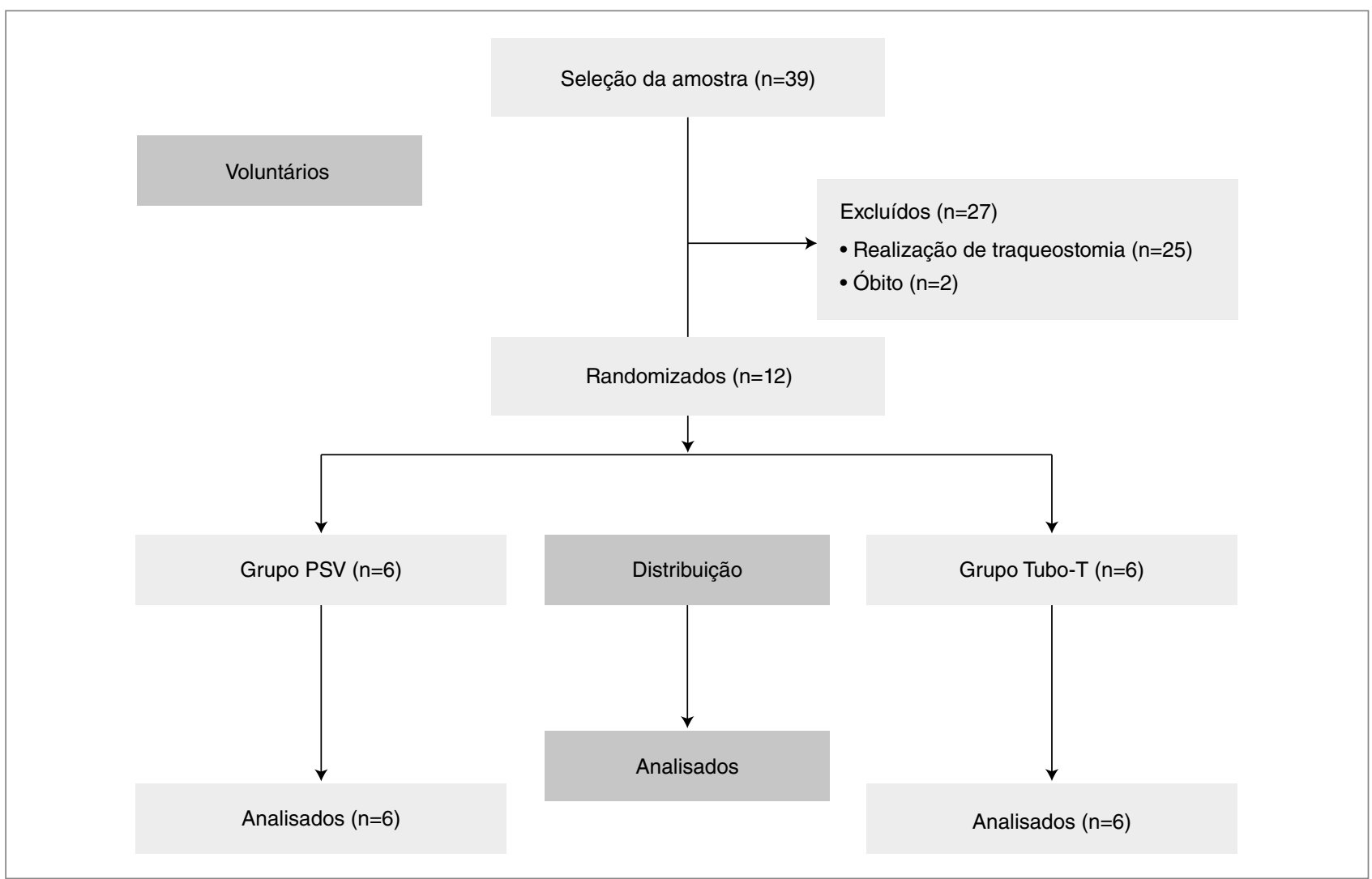

Figura 1: Fluxograma do processo de composição da amostra. 
A tabela 1 demonstra a caracterização da amostra estudada nos grupos PSV e Tubo T de acordo com sexo, idade, tipo de tratamento do TCE, causa do acidente e lesões associadas (Tabela 1).

No que se refere à comparação intragrupo do estresse oxidativo, observou-se aumento crescente das médias de áreas de descontinuidade da matriz extraceluar, no entanto, estatisticamente não significantes: Grupo PSV ( $\mathrm{p}=0.98)$ e Grupo Tubo-T: $(\mathrm{p}=0.88)$, indicando aumento do estresse oxidativo ${ }^{12}$.

Quando realizada a comparação intergrupos dos quatro momentos de avaliação do estresse oxidativo, não se observou alterações estatisticamente significativas como demonstrado na Tabela 2.

Foi realizada ainda a correlação entre parâmetros de FiO2, $\mathrm{PEEP}$ e relação $\mathrm{PaO} 2 / \mathrm{FiO} 2$ com o estresse oxidativo. No entanto, para a amostra estudada, não foi encontrada correlação estatisticamente significativa entre esses parâmetros com a descontinuidade da matriz extraceluar na interrupção da ventilação mecânica tanto no método utilizando PSV quanto no Tubo-T (Tabela 3).

No que tange ao tempo de intubação orotraqueal, correlacionou-se o tempo de ventilação mecânica com o estresse oxidativo no ultimo momento de avaliação. Verificou-se aumento estatisticamente significativo da área de descontinuidade da matriz extracelular, indicando correlação entre o aumento do estresse oxidativo com a quantidade de dias sob ventilação mecânica. O grupo PSV permaneceu em média $12,33 \pm 2,4$ dias sob ventilação mecância, tendo estresse oxidativo de 29,02 $\pm 12,75$ (grau III/moderado) $-\mathrm{r}=0,8, \mathrm{p}=0,01$. O grupo Tubo T permaneceu em ventilação mecânica em média $12,83 \pm 2,2$ dias, tendo estresse oxidativo de 31,03 $\pm 5,41$ (grau IV/grave)- $r=0,9, p=0,07$.

\section{DISCUSSÃO}

Neste estudo, foram analisados de forma longitudinal e randomizada os níveis de estresse oxidativo em vítimas de TCE no processo de interrupção da ventilação mecânica por meio da extubação nos métodos PSV e Tubo-T. De modo geral, os 12 participantes da pesquisa não apresentaram diferenças estatisticamente significativas quanto aos níveis de estresse. No entanto, para a amostra estudada, as áreas de descontinuidade da matriz extracelular tenderam a aumentar com o tempo de ventilação mecânica.

Verificou-se nesta pesquisa uma importante especificidade dos pacientes neurocríticos: grande parte dos pacientes foram excluídos do grupo PSV e Tubo-T, pois evoluíram para cânula de traqueostomia. Nesse sentido, um estudo ${ }^{13}$ relatou que em pacientes neurológicos, a TQT é indicada em cerca de 33\% dos casos. Nesse contexto, Como vantagens da TQT, podem ser consideradas a segurança quanto a manutenção da via aérea pérvea, não ocorrência de auto-extubação, a proteção da laringe, facilidade para mobilização do paciente e comunicação oral, além de poder reduzir o tempo de $\mathrm{VM}$, reduzir o trabalho respiratório, com consequentemente facilitação do desmame da VM, e utilização de menores doses de sedação e analgesia ${ }^{14,15}$.

Em contrapartida, compreende-se também os riscos e desvantagens que a traqueostomia pode acarretar. Estudos apontam a compressão exercida na região de pescoço durante a dilatação da traquéia e possível redução do fluxo venoso jugular, bem como

Tabela 2: Análise intergrupos das médias e desvio padrão das áreas de descontinuidade da matriz extracelular da análise de estresse oxidativo nos grupos PSV e Tubo-T em cada etapa de coleta.

\begin{tabular}{|c|c|c|c|}
\hline Variável & Grupo PSV & Grupo Tubo T & $p$ \\
\hline $24 \mathrm{~h}$ & $\begin{array}{c}26,92 \pm 5,08 \\
\text { Grau III (moderado) }\end{array}$ & $\begin{array}{c}26,86 \pm 13,89 \\
\text { Grau III (moderado) }\end{array}$ & $p=0,29$ \\
\hline Pré teste & $\begin{array}{c}27,8104 \pm 6,28 \\
\text { Grau III (moderado) }\end{array}$ & $\begin{array}{c}29,32 \pm 5,16 \\
\text { Grau III (moderado) }\end{array}$ & $\mathrm{p}=0,18$ \\
\hline $15^{\prime}$ de teste & $\begin{array}{c}27,61 \pm 11,58 \\
\text { Grau III (moderado) }\end{array}$ & $\begin{array}{c}30,09 \pm 10,02 \\
\text { Grau IV (grave) }\end{array}$ & $p=0,30$ \\
\hline Após o teste & $\begin{array}{c}29,02 \pm 12,75 \\
\text { Grau III (moderado) }\end{array}$ & $\begin{array}{c}31,03 \pm 5,41 \\
\text { Grau IV (grave) }\end{array}$ & $p=0,27$ \\
\hline
\end{tabular}

PSV: grupo em interrupção da ventilação mecânica pelo método pressão de suporte; Tubo-T: grupo em interrupção da ventilação mecânica pelo método Tubo -T. VM: ventilação mecânica, $p<0,05$.

Tabela 1: caracterização da amostra estudada nos grupos PSV e Tubo-T de acordo com sexo, idade, tratamento proposto, causa acidental e lesões associadas.

\begin{tabular}{|c|c|c|c|c|}
\hline Variáveis & Grupo PSV $(n=6)$ & p & Grupo Tubo T $(n=6)$ & p \\
\hline \multicolumn{5}{|l|}{ Sexo } \\
\hline Homens & $5(83,33 \%)$ & \multirow{2}{*}{1} & $5(83,33 \%)$ & \multirow{2}{*}{1} \\
\hline Mulheres & $1(16,66 \%)$ & & $1(16,66 \%)$ & \\
\hline Idade & $39,33 \pm 12,61$ & 0,75 & $29 \pm 7,74$ & 0,74 \\
\hline \multicolumn{5}{|l|}{ Tipo de tratamento } \\
\hline Craniotomia & $3(50 \%)$ & & $1(16,66 \%)$ & \\
\hline Conservador & $3(50 \%)$ & & $5(83,33 \%)$ & \\
\hline \multicolumn{5}{|l|}{ Causa } \\
\hline Moto & $3(50 \%)$ & & $3(50 \%)$ & \\
\hline Atropelamento & $2(33,33 \%)$ & & $2(33,33 \%)$ & \\
\hline Queda & $1(16,66 \%)$ & & & \\
\hline Agressão Física & & & $1(16,66 \%)$ & \\
\hline \multicolumn{5}{|l|}{ Lesões associadas } \\
\hline Fratura em membro inferior & $1(16,66 \%)$ & & $1(16,66 \%)$ & \\
\hline Fratura em membro superior & $1(16,66 \%)$ & & $1(16,66 \%)$ & \\
\hline Trauma torácico & $1(16,66 \%)$ & & $1(16,66 \%)$ & \\
\hline Sem traumas associados & $3(50 \%)$ & & $3(50 \%)$ & \\
\hline
\end{tabular}

Grupo PSV: grupo submetido à interrupção da ventilação mecânica por pressão de suporte; Grupo Tubo-T: grupo submetido à interrupção da ventilação mecânica por Tubo T 
o risco de hipóxia ou hipercapnia; sangramento ou infecção do ostoma, pneumotórax, enfisema subcutâneo, pneumomediastino, estenose subglótica ou traqueal, entre outras; sendo mais comum a ocorrência de complicações em indivíduos que permaneceram intubados por mais de 14 dias $^{14-17}$.

No contexto da variável idade, verificou-se que a maioria das vítimas foram adultos jovens, sendo os homens os mais acometidos por TCE. Isto pode ser justificado principalmente pelos acidentes de transito na vida adulta, envolvendo motos, carros e/ou atropelamentos. Em um estudo que abrangeu todo o território brasileiro os homens formaram $81,5 \%$ da amostra. O estudo considerou as internações por TCE de 2008 a 2012 e observou uma taxa de hospitalização 3,5 vezes maior em homens, sendo o período de hospitalização e a taxa de mortalidade maiores, devido a maior gravidade no sexo masculino ${ }^{18}$.

No que se refere à comparação intragrupo e intergrupo do estresse oxidativo verificou-se médias crescentes de descontinuidade da matriz extracelular, porém sem diferenças estatisticamente significativas tanto no grupo PSV, quanto no grupo Tubo-T, indicando que, para a amostra estudada, o estresse oxidativo não está relacionado diretamente com o tipo de método de interrupção da ventilação mecânica. No entanto, os níveis elevados de estresse oxidativo nas primeiras avaliações podem apresentar maior associação com outros fatores que favorecem a produção de $\mathrm{ERO}$, como o trauma e ventilação mecânica ${ }^{19}$.

Nesse sentido, um estudo ${ }^{19}$ utilizando o método TBARS para detectar substancias que reagem com o ácido tiobarbitúrico, demonstrou alteração significativa do estresse oxidativo nas primeiras $24 \mathrm{~h}$ do TCE, uma vez que em pacientes com injúria cerebral, o TBARS pode determinar uma condição de aumento de peroxidação lipídica. Outro aspecto importante a se considerar é a presença de hemorragias intraparenquimatosas ou subaracnóidea nos pacientes, as quais poderiam disponibilizar íon ferroso $\left(\mathrm{Fe}^{2+}\right)$ presente no complexo heme das hemoglobinas para interagir com ânions superóxidos levando a formação de radical hidroxila $\left(\mathrm{OH}^{-}\right)$, cuja produção marca a irreversibilidade do estresse oxidativo e um maior dano a biomacromoléculas.
Corroborando ainda com esses pressupostos, em um modelo experimental animal realizado com ratos adultos, um grupo de animais foi exposto à ventilação mecânica (VM) por 12 horas e compararam ao grupo controle (não expostos), concluiu-se que a ventilação mecânica promoveu desequilíbrio das principais defesas antioxidantes do diafragma, contribuindo para o dano na musculatura inspiratória ${ }^{6,20}$. Outro estudo com ratos expostos à VM por 18 horas evidenciou diminuição de miofibrila muscular em todos os tipos de fibras diafragmáticas, além de oxidação de proteínas e peroxidação lipídica ${ }^{6,21-23}$.

Além disso, embora não tenham sido objetos de estudo nesta pesquisa, fatores exógenos moduladores do EOx também podem influenciar o equilíbrio oxidativo, bem como ter contribuído com os níveis elevados de descontinuidade da matriz extracelular da amostra da pesquisa. Em estudo de revisão ${ }^{24}$ assinala que o fumo tem relação com a alteração dos marcadores da peroxidação lipídica, oxidação das proteínas e aumento dos níveis plasmáticos dos grupos carbonila. Ressaltando ainda que os níveis plasmáticos de vitamina $\mathrm{C}$ e $\mathrm{E}$, compostos vitamínicos potencialmente antioxidantes, encontram-se reduzidos em indivíduos fumantes.

Outro fator exógeno que apresenta influência sobre o EOx é o álcool, os efeitos podem ser diretos ou mediados por metabólitos secundários, sendo importante sua ação sobre a redução dos níveis plasmáticos ou séricos dos antioxidantes dietéticos, entre eles: $\boldsymbol{\alpha}$-tocoferol, ácido ascórbico e selênio. Ademais, o metabolismo do etanol pode estar diretamente envolvido na produção de ERO, bem como na depleção dos componentes do sistema antioxidante e aumento nos níveis de marcadores específicos, especialmente malondialdeído ${ }^{24}$.

No que se refere aos parâmetros ventilatórios, tais como PEEP, FiO2 e índice de oxigenação, não foi encontrada correlação estatisticamente significativa com o estresse oxidativo, provavelmente pelo baixo número amostral desta pesquisa. No entanto, quando correlacionado o tempo de ventilação mecânica com o estresse oxidativo, esta pesquisa indicou que quanto maior o tempo de

Tabela 3: correlação entre parâmetros de FiO2, PEEP e relação $\mathrm{FiO} / \mathrm{PaO} 2$ com o estresse oxidativo nos métodos de interrupção da ventilação mecânica - PSV e Tubo-T; na admissão e antes do teste.

\begin{tabular}{|c|c|c|c|}
\hline \multicolumn{4}{|c|}{ PSV } \\
\hline Variável & Média e DP & $r$ & $\mathbf{p}$ \\
\hline PEEP incial vs EOx $24 \mathrm{~h}$ & $8,1 \pm 1,8$ vs $26,92 \pm 5,08$ & 0,3 & 0,4 \\
\hline PEEP Final vs EOx pré teste & $5,8 \pm 1,1$ vs $27,81 \pm 6,2$ & $-0,2$ & 0,5 \\
\hline FiO2 incial vs EOx $24 \mathrm{~h}$ & $55 \pm 16,46$ vs $26,92 \pm 5,08$ & 0,5 & 0,2 \\
\hline FiO2 final vs EOx Pré teste & $30,5 \pm v s 27,81 \pm 6,2$ & $-0,1$ & 0,8 \\
\hline $\mathrm{PaO} 2$ incial vs $\mathrm{EO} \times 24 \mathrm{~h}$ & $269,4 \pm 48$ vs $26,92 \pm 5,08$ & $-0,6$ & 0,1 \\
\hline $\mathrm{PaO} 2$ final vs EOx pré teste & $384,0 \pm 28,5$ vs $27,81 \pm 6,2$ & 0,2 & 0,6 \\
\hline \multicolumn{4}{|c|}{ TUBO $-\mathrm{T}$} \\
\hline Variável & Média e DP & $\mathbf{r}$ & $\mathbf{p}$ \\
\hline PEEP incial vs EOx $24 \mathrm{~h}$ & $7,5 \pm 0,5$ vs $26,86 \pm 13,89$ & 0,1 & 0,7 \\
\hline PEEP Final vs EOx pré teste & $5,5 \pm 0,5$ vs $29,32 \pm 5,1$ & $-0,09$ & 0,8 \\
\hline $\mathrm{FiO} 2$ incial vs EOx $24 \mathrm{~h}$ & $50,8 \pm 1,07$ vs $26,86 \pm 13,89$ & 0,03 & 0,9 \\
\hline FiO2 final vs EOx Pré teste & $28,3 \pm 2,5$ vs $29,32 \pm 5,1$ & 0,6 & 0,1 \\
\hline $\mathrm{PaO} 2$ incial vs $\mathrm{EO} \times 24 \mathrm{~h}$ & $283,5 \pm 41$ vs $26,86 \pm 13,89$ & 0,05 & 0,9 \\
\hline $\mathrm{PaO} 2$ final vs EOx pré teste & $372,5 \pm 35,6$ vs $29,32 \pm 5,1$ & $-0,7$ & 0,1 \\
\hline
\end{tabular}

PSV: grupo em interrupção da ventilação mecânica pelo método pressão de suporte; Tubo-T: grupo em interrupção da ventilação mecânica pelo método Tubo -T; E.O: estresse oxidativo; PEEP: pressão positiva ao final da expiração; FiO2: fração inspirada de oxigênio. p<0,05. 
ventilação mecânica de pacientes vítimas de TCE, maior a área de descontinuidade da matriz extracelular.

Dessa forma, a literatura expõe que a manutenção das trocas gasosas em pacientes sob ventilação mecânica invasiva pode ocasionar efeitos deletérios ao organismo. Pacientes com pressão positiva elevada com altos níveis de fração inspirada de oxigênio (FIO2) geram estresse oxidativo à níveis em que mecanismos antioxidantes tornam-se ineficientes. Assim sendo, ajustes inadequados nos parâmetros ventilatórios podem acarretar, por exemplo, hiperóxia culminando com os elevados níveis de estresse oxidativo².

$\mathrm{Na}$ presente pesquisa, os parâmetros ventilatórios não apresentaram forte relação com o estresse oxidativo, porém fatores como o trauma, as lesões secundárias, as drogas e o uso da ventilação mecânica invasiva podem ter contribuído para os níveis elevados de estresse oxidativo na amostra estudada. Durante o trauma, a laceração, a hemorragia e a contusão, podem ser responsáveis por muitos dos danos cerebrais. Além disso, o mecanismo secundário, como cascatas de reações bioquímicas complexas e alterações fisiopatológicas, tem apresentado grande destaque no que se refere ao impacto substancial no prognóstico dos pacientes com traumatismo crânio-encefálico ${ }^{25}$.

No TCE há um aumento considerável na produção de radicais livres, apoiando a idéia de que o estresse oxidativo desempenha um papel decisivo nesse trauma. Um dos mecanismos secundários que possivelmente exerce função chave na fisiopatologia que segue o traumatismo craniano grave é a geração excessiva espécies reativas de oxigênio por atividade de neutrófilos, células endoteliais e possivelmente neurônios e células da glia. As membranas lipídicas no cérebro contêm altos níveis de ácidos graxos poliinsaturados e são, portanto, particularmente sensíveis à oxidação. Há evidências em estudos com animais, que, além de certo grau de dano da barreira hemato-encefálica (BHE) provocado pelo mecanismo primário do trauma, a formação de ERO seguida de peroxidação lipídica causa ainda mais danos e quebra da BHE, e finalmente os limites do dano tecidual são estendidos para além da área inicial do impacto por esses mecanismos secundários ${ }^{19,26-28}$.

Outras fontes de radicais livres são a excitotoxicidade mediada pelo glutamato, que inclui a formação de bradicinina. Esta citocina pode ativar a fosfolipase A2, liberando ácido araquidônico que pode servir como fonte de radicais livres ${ }^{27}$. $\mathrm{O}$ ácido araquidônico também pode facilitar a atividade da NADPH oxidase, aumentando ainda mais a produção de ERO. Além de aumentar a produção de ácido araquidônico a partir de fosfolípidos de membrana, a bradicinina pode induzir a produção de radicais livres causando uma sobrecarga de $\mathrm{Ca} 2$ +. Outra fonte de ERO no TCE pode ser macrófagos/microglia e neutrófilos ativados como parte de um processo inflamatório desencadeado pela lesão inicial ${ }^{29,30}$.
Vale ressaltar que a presente pesquisa apresentou limitações relacionadas com a especificidade do manejo do paciente neurocrítico. A amostra para tratamento estatístico foi reduzida devido à exclusão de pacientes da pesquisa em decorrência da realização de traqueostomia, não sendo possível a extubação desses pacientes. Sugere-se às pesquisas futuras que seja incluido um maior número de coletas, a fim de verificar o comportamento do estresse oxidativo também em pacientes traqueostomizados.

Outra limitação do estudo acerca da análise do estresse oxidativo é que não foi coletada a variável antes da entrada na ventilação mecânica, pois pela gravidade do caso geralmente o paciente já era admitido e colocado em ventilação mecânica. Desta forma, não foi possível identificar se este paciente já possuía o estresse oxidativo aumentado no momento da internação hospitalar, bem como não foi coletado o momento em que o paciente estava em ar ambiente.

Além disso, o método utilizado nesta pesquisa para avaliação do estresse oxidativo ainda encontra-se pouco difundido, dificultando a fundamentação teórica para a sua utilização, bem como a comparação e discussão com outros autores. Este método, por tratar-se de uma metodologia de análise do estresse oxidativo de forma indireta, não permite identificar e diferenciar os fatores oxidativos e antioxidativos, sendo somente possível identificar o efeito do estresse oxidativo na formação da matriz extracelular da formação do coágulo.

Frisa-se ainda a importância de pesquisas que fomentem o entendimento do estresse oxidativo em seres humanos, uma vez que grande parte dos estudos são realizados em animais; e assim podendo vislumbrar um maior conhecimento à cerca do estresse oxidativo a fim de nortear o manejo clínico e terapêutico de vítimas de TCE.

Desta forma conclui-se que, em relação ao objeto central de estudo desta pesquisa, o estresse oxidativo, verificou-se que não houve alterações estatisticamente significativas na descontinuidade da matriz extracelular durante os testes de respiração espontânea no método PSV e Tubo-T, tanto nas comparações intragrupos, quanto intergrupos.

No contexto da ventilação mecânica, não se verificou correlação entre o estresse oxidativo com os níveis de PEEP, FiO2 e relação $\mathrm{FiO} 2 / \mathrm{PaO} 2$, provavelmente devido ao número amostral reduzido. No entanto, evidenciou-se que o nível de estresse oxidativo encontrava-se elevado em vítimas de TCE logo nas primeiras avaliações, indicando que o trauma, na amostra estudada, pode ter sido fator principal para o aumento da descontinuidade da matriz extracelular.

Além disso, os resultados apontaram que quanto maior o tempo de ventilação mecânica, maior a área de descontinuidade da matriz extracelular, porém não havendo diferença no comportamento do estresse oxidativo entre os métodos de interrupção da ventilação mecânica PSV e Tubo-T.

\section{REFERÊNCIAS}

1. Lima MVC, Guimarães RMO, Silva GPF, Mont'Alverne DGB. Perfil clínico e desmame ventilatório de pacientes acometidos por traumatismo crânio-encefálico. Rev Neurocienc. 2012;20(3):354-9
2. Mazullo Filho JBP, Bona S, Rosa DP, Silva FG, Forgiarini Junior LA, Dias AS, et al. Os efeitos da ventilação mecânica no estresse oxidativo. Rev Bras Ter Intensiva. 2012;24(1):23-9. http://dx.doi.org/10.1590/S0103-507X2012000100004 
3. Ribeiro JS, Baldan CS, Masson IFB, Esteves Júnior I, Bernardo K, Civile VT. Análise da variação percentual do índice de respiração rápida e superficial (IRRS) no desmame ventilatório. J Health Sci Inst. 2013;31(2):205-9.

4. Lopes JSC, Jesus PNLG, Machado TO, Reis HFC. Preditores de falha de extubação em unidade de terapia intensiva. Rev Pesq Fisioter. 2016;6(2):179-88. http://dx.doi.org/2238-2704rpf.v6i2.889

5. Freitas ERFS, Favarão C, Chivalki EP, Pessoa JSF. Ventilação mecânica em pacientes com síndrome da angústia respiratória aguda. UNOPAR Cient. 2007;9(1):53-60.

6. Verona C, Benfato MS, Teixeira C. Associação da ventilação mecânica e seu desmame com o estresse oxidativo. Dissertação (Mestrado) Universidade Federal do Rio Grande do Sul. Porto Alegre: 2013.

7. Pinho WL, Silva APR. Efeitos do exercício físico sobre a formação de espécies reativas de oxigênio e os compostos antioxidantes da dieta. Rev Bras Nutr Esportiva. 2013;7(37):77-87.

8. Davidovich N, DiPaolo BC, Lawrence GG, Chhour P, Yehya N, Margulies SS. Cyclic Stretch-Induced Oxidative Stress Increases Pulmonary Alveolar Epithelial Permeability. Am J Respir Cell Mol Biol. 2013;49(1):156-64. http://dx.doi.org/10.1165/rcmb.2012-0252OC

9. Carlos SP, Dias AS, Forgiarini Júnior LA, Patrício, PD, Graciano T, Nesi RT, et al. Dano oxidativo induzido por exposição a fumaça de cigarro em camundongos: impacto sobre o pulmão e o músculo diafragma. J Bras Pneumol. 2014;40(4):411-20.

10. Tasaka S, Amaya F, Hashimoto S, Ishizaka A. Roles of oxidants and redox signaling in the pathogenesis of acute respiratory distress syndrome. Antiox Redox Signal. 2008;10(4):739-54. http://dx.doi.org/10.1089/ars.2007.1940

11. Prado FP, Paludetto DRB, Bachur CAK, Freitas RAL, Zaia JE, Barros Neto TL, et al. Estresse oxidativo no plasma sanguíneo de indivíduos submetidos ao esforco físico agudo seguido de crioimersão corporal. Fisioter Pesqui. 2012;19(3):215-21. http://dx.doi.org/10.1590/S1809-29502012000300005

12. Olszewer E. Microscopia óptica como método de medida de radicais livres. 2ed. São Paulo: Ícone, 2001.

13. Tomicic FV, Andresen HM. Ventilación mecánica en el paciente con lesión cerebral aguda. Rev Med Chile. 2011;139(3):382-90. http://dx.doi.org/10.4067/S0034-98872011000300016

14. Shamim MS, Qadeer M, Murtaza G, Enam SA, Faroogi NB. Emergency department predictors of tracheostomy in patients with isolated traumatic brain injury requiring; emergency cranial decompression. J Neurosurg. 2011;115(5):1007-12. http://dx.doi.org/10.3171/2011.7.JNS101829

15. Lazaridis C, DeSantis SM, McLawhorn M, Krishna V. Liberation of neurosurgical patients from mechanical ventilation and tracheostomy in neurocritical care. J Crit Care. 2012;27(8):417.e1-8. http://dx.doi.org/10.1016/j.jcrc.2011.08.018

16. Pinheiro BV, Tostes RO, Brum Cl, Carvalho, EV, Pinto SPS, Oliveira JCA. Traqueostomia precoce versus traqueostomia tardia em pacientes com lesão cerebral aguda grave. J Bras Pneumol. 2010;36(1):84-91. http://dx.doi.org/10.1590/S1806-37132010000100014

17. Huang $\mathrm{H}$, Li Y, Ariani F, Chen X, Lin J. Timing of tracheostomy in critically ill patients: a meta-analysis. PLoS One. 2014;(3):e92981. http://dx.doi.org/10.1371/journal.pone.0092981
18. Magalhães ALG, Souza LC, Faleiro RM, Teixeira AL, Miranda AS. Epidemiologia do traumatismo cranioencefálico no Brasil. Rev Bras Neurol. 2017:53(2):15-22.

19. Bertotti MM. Associação entre os níveis plasmáticos de marcadores de estresse oxidativo e mortalidade de pacientes com trauma craniano grave. Monografia (Conclusão de Curso). Universidade Federal de Santa Catarina. Florianópolis: 2009; p. 5-7

20. Falk DJ, DeRuisseau KC, Van Gammeren DL, Deering MA, Kavazis AN, Powers SK. Mechanical ventilation promotes redox status alterations in the diaphragm. J Appl Physiol. 2006;101(4):1017-24

http://dx.doi.org/10.1152/japplphysiol.00104.2006

21. Lecuona E, Sassoon CS, Barreiro E. Lipid overload: trigger or consequence of mitochondrial oxidative stress in ventilatorinduced diaphragmatic dysfunction? Am J Respir Crit Care Med. 2012;186(11)1074-6.

http://dx.doi.org/10.1164/rccm.201209-1735ED

22. Picard M, Jung B, Liang F, Azuelos I, Hussain S, Goldberg P, et al. Mitochondrial dysfunction and lipid accumulation in the human diaphragm during mechanical ventilation. Am J Respir Crit Care Med. 2012;186(11):1140-9.

http://dx.doi.org/10.1164/rccm.201206-0982OC

23. Powers SK, Wiggs MP, Duarte JA, Zergeroglu AM, Demirel HA. Mitochondrial signaling contributes to disuse muscle atrophy. Am J Endocrinol Metab. 2012;303(1):E31-9.

http://dx.doi.org/10.1152/ajpendo.00609.2011

24. Barbosa KBF, Costa NMB, Alfenas RCG, Paula SO, Minim VPR, Bressan J. Estresse oxidativo: conceito, implicações e fatores modulatórios. Rev Nutr. 23(4):629-43.

http://dx.doi.org/10.1590/\$1415-52732010000400013

25. Murray GD, Butcher I, McHugh GS, Lu J, Mushkudiani NA, Maas $\mathrm{Al}$, et al. Multivariable prognostic analysis in traumatic brain injury: results from the IMPACT study. J Neurotrauma. 2007;24(2):329-37. http://dx.doi.org/10.1089/neu.2006.0035

26. Arent AM, Souza LF, Walz R, Dafre AL. Perspectives on molecular biomarkers of oxidative stress and antioxidant strategies in traumatic brain injury. Bio Med Res Int. 2014;(2014):1-18. http://dx.doi.org/10.1155/2014/723060

27. Mckee AC, Daneshvar DH. The neuropathology of traumatic brain injury. Handb Clin Neurol. 2015;127:45-66. http://dx.doi.org/10.1016/B978-0-444-52892-6.00004-0

28. Lozano D, Gonzales-Portillo GS, Acosta S, de la Pena I, Tajir $N$, Kaneko $Y$, et al. Neuroinflammatory responses to traumatic brain injury: etiology, clinical consequences, and therapeutic opportunities. Neuropsychiatr Dis Treat. 2015;11:97-106. http://dx.doi.org/10.2147/NDT.S65815

29. Faller S, Seiler R, Donus R, Engelstaedter H, Hoetzel A, Spassov SG. Pre-and posttreatment with hydrogen sulfide prevents ventilator-induced lung injury by limiting inflammation and oxidation. PloS One. 2017;12(10):0186532. http://dx.doi.org/10.1371/journal.pone.0176649

30. Haddad SH, Arabi YM. Critical care management of severe traumatic brain injury in adults. Scand J Trauma Resusc Emerg Med. 2012;20:12

http://dx.doi.org/10.1186/1757-7241-20-12 